\title{
LIMIT THEOREMS FOR BIVARIATE EXTREMES OF NON-IDENTICALLY DISTRIBUTED RANDOM VARIABLES
}

Abstract. The limit behaviour of the extreme order statistics arising from $n$ two-dimensional independent and non-identically distributed random vectors is investigated. Necessary and sufficient conditions for the weak convergence of the distribution function (d.f.) of the vector of extremes, as well as the form of the limit d.f.'s, are obtained. Moreover, conditions for the components of the vector of extremes to be asymptotically independent are studied.

1. Introduction. Only in the last few years are general results on order statistics from non-identically but independently distributed random variables (r.v.'s) coming out. However, most of these results concern recurrence relations for the d.f. (see, e.g., Cao and West, 1997) or for the single and product moments of order statistics (see, e.g., Balakrishnan, 1994, and references therein). The work on the asymptotic behaviour of order statistics from non-identical r.v.'s is still limited. An earlier result and perhaps the most important one for this problem is due to Mejzler (1949-1956). Under a natural uniformity assumption (that the initial suitably normalized r.v.'s should, in some sense, be individually negligible in the limit), Mejzler proved that a nondegenerate d.f. $\Phi_{1}(x)$ is the limiting distribution of a suitably normalized maximum for some sequence of independent r.v.'s if, and only if, either (i) $\log \Phi_{1}(x)$ is concave, or (ii) $\omega=\sup \left\{x: \Phi_{1}(x)<1\right\}<\infty$ and $\log \Phi_{1}\left(\omega-e^{-x}\right), x>0$, is concave, or finally (iii) $\alpha=\inf \left\{x: \Phi_{1}(x)>0\right\}$ is finite and $\log \Phi_{1}\left(\alpha+e^{x}\right), x>0$, is concave. Mejzler and Weissman (1969) extended this result to $k$ th extremes. They proved that the d.f. of the $k$ th extremes (suitably normalized), under the uniformity assumption, converges

2000 Mathematics Subject Classification: Primary 60F05; Secondary 62E15, 62E20, $62 \mathrm{G} 30$.

Key words and phrases: order statistics, weak convergence, bivariate extremes. 
weakly to a nondegenerate limit d.f. $\Phi_{k}(x)$ if, and only if, the d.f. of the maximum converges weakly to the d.f. $\Phi_{1}(x)$. Balkema et al. (1993) (Theorem A.1) proved Mejzler's result on limit distributions for maxima of a sequence of independent r.v.'s, replacing the uniformity assumption by less restrictive conditions (imposed on the normalizing constants). Additional interesting results on independent sequences were given in Weissman (1975a, b), Tiago de Oliveira (1976), Mucci (1977) and de Hann and Verkade (1985). A survey of recent developments can be found in Galambos (1987).

The main aim of this paper is to study the limit behaviour of the order statistics of bivariate independent non-identically distributed r.v.'s. Namely, consider $n$ two-dimensional independent random vectors $\underline{X}_{j}=$ $\left(X_{1 j}, X_{2 j}\right), j=1, \ldots, n$, with the respective d.f.'s $F_{j}(\underline{x})=F_{j}\left(x_{1}, x_{2}\right)=$ $P\left(X_{1 j} \leq x_{1}, X_{2 j} \leq x_{2}\right), j=1, \ldots, n$. The order statistics of the $t$ th components are

$$
X_{t, 1: n} \leq X_{t, 2: n} \leq \ldots \leq X_{t, n: n}, \quad t=1,2 .
$$

The main object of this paper is to investigate the limiting distribution of the random vector $\underline{Z}_{k, k^{\prime}: n}=\left(X_{1, n-k+1: n}, X_{2, n-k^{\prime}+1: n}\right)$, where $k$ and $k^{\prime}$ are constants. Necessary and sufficient conditions under which the d.f. of $\underline{Z}_{k, k^{\prime}: n}$ converges weakly to a nondegenerate limit d.f., as well as the form of this limit, will be obtained in Theorem 2.1. Necessary and sufficient conditions (in terms of the original sequence of d.f.'s $\left\{F_{j}(\underline{x})\right\}$ ) for the components of $\underline{Z}_{k, k^{\prime}: n}$ to be asymptotically independent are derived in Theorem 2.2. The problem can also be stated in terms of $\underline{W}_{k, k^{\prime}: n}=\left(X_{1, k: n}, X_{2, k^{\prime}: n}\right)$ and $\underline{V}_{k, k^{\prime}: n}=\left(X_{1, k: n}, X_{2, n-k^{\prime}+1: n}\right)$ by turning respectively to $\left(-X_{1 j},-X_{2 j}\right)$ and $\left(-X_{1 j}, X_{2 j}\right), j=1, \ldots, n$.

The results of this paper can be applied to many natural problems, e.g., project scheduling by PERT technique. In this practical problem we assume a large number $n$ of different activities, each of them has a random duration and a random cost (which usually depends on the duration). In many cases, the durations of different activities are non-identically distributed r.v.'s, and so are the costs of different activities. On the other hand the duration and the cost of each activity are in general dependent r.v.'s. Therefore, we get a sequence of $n$ two-dimensional non-identical r.v.'s $\left\{\underline{X}_{j}\right\}=\left\{\left(X_{1 j}, X_{2 j}\right)\right\}, j=$ $1, \ldots, n$, where $X_{1 j}$ and $X_{2 j}$ denote respectively the duration and the cost of the $j$ th activity. It is well known that the vectors $\underline{Z}_{1,1: n}, \underline{W}_{1,1: n}$ and $\underline{V}_{1,1: n}$ for this sequence play a major role in the investigation of the above stated problem.

Throughout this paper the following conventions and notations will be adopted. For numerical vectors $\underline{x}=\left(x_{1}, x_{2}\right)$, the components are signified by a subscript. Basic arithmetical operations are always meant component-wise. Thus $\underline{x} \leq \underline{y}$ means $x_{t} \leq y_{t}, t=1,2$. Further, $\underline{x} \pm \underline{y}=\left(x_{1} \pm y_{1}, x_{2} \pm y_{2}\right)$, 
$\underline{x} \underline{y}=\left(x_{1} y_{1}, x_{2} y_{2}\right)$ and $\underline{x} / \underline{y}=\left(x_{1} / y_{1}, x_{2} / y_{2}\right)$. The special vectors $\underline{0}=(0,0)$ and $\underline{\infty}=(\infty, \infty)$ will be used. Let $G_{j}(\underline{x})=P\left(\underline{X}_{j}>\underline{x}\right)$ be the survival function of $F_{j}(\underline{x}), j=1,2, \ldots$, and let $\Phi_{., k^{\prime}: n}\left(x_{2}\right), \Phi_{k,: n}\left(x_{1}\right), F_{1 j}\left(x_{1}\right)$, $F_{2 j}\left(x_{2}\right), G_{1 j}\left(x_{1}\right)=1-F_{1 j}\left(x_{1}\right)$ and $G_{2 j}\left(x_{2}\right)=1-F_{2 j}\left(x_{2}\right)$ be the marginal d.f.'s and the marginal survival functions of $\Phi_{k, k^{\prime}: n}(\underline{x})=P\left(\underline{Z}_{k, k^{\prime}: n} \leq \underline{x}\right)$, $F_{j}(\underline{x})$ and $G_{j}(\underline{x}), j=1,2, \ldots$, respectively. Finally, for any sequence of suitable normalizing constants $\underline{a}_{n}=\left(a_{1 n}, a_{2 n}\right)>\underline{0}$ and $\underline{b}_{n}=\left(b_{1 n}, b_{2 n}\right)$, write $\underline{Z}_{k, k^{\prime}: n}^{\star}=\left(\underline{Z}_{k, k^{\prime}: n}-\underline{b}_{n}\right) / \underline{a}_{n}, \Phi_{k, k^{\prime}: n}^{\star}(\underline{x})=\Phi_{k, k^{\prime}: n}\left(\underline{a}_{n} \underline{x}+\underline{b}_{n}\right), F_{j: n}^{\star}(\underline{x})=$ $F_{j}\left(\underline{a}_{n} \underline{x}+\underline{b}_{n}\right), G_{j: n}^{\star}(\underline{x})=G_{j}\left(\underline{a}_{n} \underline{x}+\underline{b}_{n}\right), F_{t j: n}^{\star}\left(x_{t}\right)=F_{t j}\left(a_{t n} x_{t}+b_{t n}\right), G_{t j: n}^{\star}\left(x_{t}\right)=$ $G_{t j}\left(a_{t n} x_{t}+b_{t n}\right), t=1,2, \Phi_{., k^{\prime}: n}^{\star}\left(x_{2}\right)=\Phi_{., k^{\prime}: n}\left(a_{2 n} x_{2}+b_{2 n}\right)$ and $\Phi_{k,: n}^{\star}\left(x_{1}\right)=$ $\Phi_{k,: n}\left(a_{1 n} x_{1}+b_{1 n}\right)$.

We conclude this section with a theorem which is a combination of the results of Juncosa (1949), Sec. 3 (see also Galambos, 1987) and Mejzler and Weissman (1969), Theorem 4.1. This theorem will be needed in what follows.

THEOREM 1.1. Assume that, for suitable normalizing constants $a_{1 n}>0$, $b_{1 n}\left(\right.$ resp. $\left.a_{2 n}>0, b_{2 n}\right)$,

$$
\begin{aligned}
\delta_{1: n} & =\max _{1 \leq j \leq n} G_{1 j: n}^{\star}\left(x_{1}\right) \rightarrow 0 \text { as } n \rightarrow \infty \\
\text { (resp. } \delta_{2: n} & \left.=\max _{1 \leq j \leq n} G_{2 j: n}^{\star}\left(x_{2}\right) \rightarrow 0 \text { as } n \rightarrow \infty\right) .
\end{aligned}
$$

Then $\Phi_{k,: n}^{\star}\left(x_{1}\right)$ (resp. $\left.\Phi_{., k^{\prime}: n}^{\star}\left(x_{2}\right)\right)$ converges weakly to a nondegenerate d.f. $\Phi_{k, .}\left(x_{1}\right)\left(\right.$ resp. $\left.\Phi_{., k^{\prime}}\left(x_{2}\right)\right)$ if , and only if, for all $x_{1}$ (resp. $\left.x_{2}\right)$ for which $\Phi_{k, .}\left(x_{1}\right)>0$ (resp. $\left.\Phi_{., k^{\prime}}\left(x_{2}\right)>0\right)$, the limit

$$
\lim _{n \rightarrow \infty} \sum_{i=1}^{n} G_{1 i: n}^{\star}\left(x_{1}\right)=h_{1}\left(x_{1}\right) \quad\left(\text { resp. } \lim _{n \rightarrow \infty} \sum_{i=1}^{n} G_{2 i: n}^{\star}\left(x_{2}\right)=h_{2}\left(x_{2}\right)\right)
$$

is finite, and the function

$$
\Phi_{k, .}\left(x_{1}\right)=\sum_{i=0}^{k-1} \frac{h_{1}^{i}\left(x_{1}\right)}{i !} e^{-h_{1}\left(x_{1}\right)}\left(\text { resp. } \Phi_{., k^{\prime}}\left(x_{2}\right)=\sum_{i=0}^{k^{\prime}-1} \frac{h_{2}^{i}\left(x_{2}\right)}{i !} e^{-h_{2}\left(x_{2}\right)}\right)
$$

is a nondegenerate d.f. The actual limit d.f. of

$$
Z_{k,: n}^{\star}=\frac{X_{1, n-k+1: n}-b_{1 n}}{a_{1 n}} \quad\left(\text { resp. } Z_{., k^{\prime}: n}^{\star}=\frac{X_{2, n-k^{\prime}+1: n}-b_{2 n}}{a_{2 n}}\right)
$$

is the one given in (1.2).

As a direct result of this theorem we see that the convergence of $\Phi_{k, .: n}^{\star}\left(x_{1}\right)$ (resp. $\left.\Phi_{., k^{\prime}: n}^{\star}\left(x_{2}\right)\right)$ for at least one fixed value of $k$ (resp. $k^{\prime}$ ) implies its convergence for all fixed values of $k$ (resp. $k^{\prime}$ ). Moreover, the possible types of the function $h_{1}\left(x_{1}\right)$ (resp. $h_{2}\left(x_{2}\right)$ ) may be determined from the above mentioned results of Mejzler (1949-1956) (since $\Phi_{1, .}\left(x_{1}\right)=e^{-h_{1}\left(x_{1}\right)}$ and $\left.\Phi ., 1\left(x_{2}\right)=e^{-h_{2}\left(x_{2}\right)}\right)$. 
2. Main results. It is easy to show that the d.f. of the vector $\underline{Z}_{k, k^{\prime}: n}$ is given by $\Phi_{k, k^{\prime}: n}(\underline{x})=P\left(\right.$ at most $k-1$ and $k^{\prime}-1$ of events $\left\{X_{1 i}>x_{1}\right\}$ and $\left\{X_{2 j}>x_{2}\right\}, i, j=1, \ldots, n$, occur respectively). Consequently, by taking into account the fact that the vectors $\left(X_{1 j}, X_{2 j}\right), j=1, \ldots, n$, are independent and by collecting terms according as $\left\{X_{1 j}>x_{1}, X_{2 j} \leq x_{2}\right\}$, or $\left\{X_{1 j}>x_{1}, X_{2 j}>x_{2}\right\}$, or $\left\{X_{1 j} \leq x_{1}, X_{2 j}>x_{2}\right\}$, or $\left\{X_{1 j} \leq x_{1}, X_{2 j} \leq x_{2}\right\}$, $j=1, \ldots, n$, we get

$$
\begin{aligned}
\Phi_{k, k^{\prime}: n}(\underline{x})= & \sum_{t=0}^{k-1} \sum_{s=0}^{k^{\prime}-1} \sum_{r=0 \vee(t+s-n)}^{t \wedge s} \sum_{\mathcal{P}_{r, s, t}} \prod_{j=1}^{t-r} P\left(X_{1 i_{j}}>x_{1}, X_{2 i_{j}} \leq x_{2}\right) \\
& \times \prod_{j=t-r+1}^{t} P\left(X_{1 i_{j}}>x_{1}, X_{2 i_{j}}>x_{2}\right) \\
& \times \prod_{j=t+1}^{t+s-r} P\left(X_{1 i_{j}} \leq x_{1}, X_{2 i_{j}}>x_{2}\right) \\
& \times \prod_{j=t+s-r+1}^{n} P\left(X_{1 i_{j}} \leq x_{1}, X_{2 i_{j}} \leq x_{2}\right) \\
= & \sum_{t=0}^{k-1} \sum_{s=0}^{k^{\prime}-1} \sum_{r=0 \vee(t+s-n)}^{t \wedge s} \sum_{\mathcal{P}_{r, s, t}}^{t-r} \prod_{j=1}^{t+s-r}\left(G_{1 i_{j}}\left(x_{1}\right)-G_{i_{j}}(\underline{x})\right) \\
& \times \prod_{j=t-r+1}^{t} G_{i_{j}}(\underline{x}) \prod_{j=t+1}^{n}\left(G_{2 i_{j}}\left(x_{2}\right)-G_{i_{j}}(\underline{x})\right) \\
& \times \prod_{j=t+s-r+1}^{n}\left(1-G_{1 i_{j}}\left(x_{1}\right)-G_{2 i_{j}}\left(x_{2}\right)+G_{i_{j}}(\underline{x})\right),
\end{aligned}
$$

where $\min (a, b)=a \wedge b, \max (a, b)=a \vee b$, and for $0 \leq s<k, 0 \leq t<k^{\prime}$ and $0 \leq r \leq t \wedge s, \mathcal{P}_{r, s, t}$ denotes the set of permutations $\left(i_{1}, \ldots, i_{n}\right)$ of $(1, \ldots, n)$ such that $i_{1}<\ldots<i_{t-r}, i_{t-r+1}<\ldots<i_{t}, i_{t+1}<\ldots<i_{t+s-r}$ and $i_{t+s-r+1}<\ldots<i_{n}$.

REMARK 2.1. In (2.1), and in what follows, we adopt the convention that the product of the sort $\prod_{i=a}^{b} A_{i}$, for any integers $a, b$ and any sequence $\left\{A_{i}\right\}$, is $A_{a} A_{a+1} \ldots A_{b}$ if $a \leq b$, and 1 if $b<a$. Therefore, if $x_{1} \rightarrow \infty$ (say) we get $G_{1 j}\left(x_{1}\right), G_{j}(\underline{x}) \rightarrow 0, j=1, \ldots, n$. We thus have $t \equiv r \equiv 0$, implying

$$
\begin{aligned}
\Phi_{., k^{\prime}: n}\left(x_{2}\right)= & \sum_{s=0}^{k^{\prime}-1} \sum_{\mathcal{P}_{s}} \prod_{j=1}^{s}\left(G_{2 i_{j}}\left(x_{2}\right)-G_{i_{j}}(\underline{x})\right) \\
& \times \prod_{j=s+1}^{n}\left(1-G_{1 i_{j}}\left(x_{1}\right)-G_{2 i_{j}}\left(x_{2}\right)+G_{i_{j}}(\underline{x})\right),
\end{aligned}
$$


where $\mathcal{P}_{s}$ is the set of permutations $\left(i_{1}, \ldots, i_{n}\right)$ of $(1, \ldots, n)$ such that $i_{1}<$ $\ldots<i_{s}$ and $i_{s+1}<\ldots<i_{n}$. Moreover, when $k=k^{\prime}=1$, we get

$$
\Phi_{1,1: n}(\underline{x})=\prod_{j=1}^{n}\left(1-G_{1 j}\left(x_{1}\right)-G_{2 j}\left(x_{2}\right)+G_{j}(\underline{x})\right)=\prod_{j=1}^{n} F_{j}(\underline{x}) .
$$

Relation (2.1) may be written in term of permanents as follows:

$$
\begin{aligned}
& \Phi_{k, k^{\prime}: n}(\underline{x})=\sum_{t=0}^{k-1} \sum_{s=0}^{k^{\prime}-1} \sum_{r=0 \vee(t+s-n)}^{t \wedge s} \frac{1}{(t-r) ! r !(s-r) !(n-t-s+r) !} \\
& \times \operatorname{Per}\left[\underline{Y}_{1 .}{ }_{t-r} \underline{Y}_{11} \quad \underline{Y}_{r} \quad \underline{Y}_{{ }_{1}}-\underline{Y}_{s-r} \quad \underline{1}_{-}-\underline{Y}_{1 .}-\underline{Y}_{n-t}+\underline{Y}_{11}+\underline{Y}_{11}\right],
\end{aligned}
$$

where $\underline{Y}_{1 .}=\left(G_{11}\left(x_{1}\right) \ldots G_{1 n}\left(x_{1}\right)\right)^{\prime}, \underline{Y}_{.1}=\left(G_{21}\left(x_{2}\right) \ldots G_{2 n}\left(x_{2}\right)\right)^{\prime}, \underline{Y}_{11}=$ $\left(G_{1}(\underline{x}) \ldots G_{n}(\underline{x})\right)^{\prime}$ and $\underline{1}$ is the $n \times 1$ column vector of ones. Moreover, if $\underline{a}_{1}, \underline{a}_{2}, \ldots$ are column vectors, then

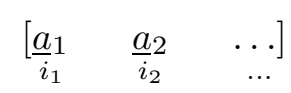

will denote the matrix obtained by taking $i_{1}$ copies of $\underline{a}_{1}, i_{2}$ copies of $\underline{a}_{2}$ and so on. Finally, in (2.2), $\operatorname{Per}(A)$ denotes the permanent of a square matrix $A$, which is defined similarly to the determinant except that all terms in the expansion have a positive sign (see Minc's book, 1978, and the survey papers of Minc, 1983, 1987). It is worth mentioning that the permanent in (2.2) is a stochastic one.

REMARK 2.2. In view of Remark 2.1, we adopt the convention that

$$
\operatorname{Per}\left[\begin{array}{lllll}
\underline{a}_{1} & \underline{a}_{2} & \cdots & \underline{a}_{j} & \cdots
\end{array}\right]
$$

is understood as

$$
\operatorname{Per}\left[\begin{array}{llllll}
\underline{a}_{1} & \underline{a}_{2} & \cdots & \underline{a}_{j-1} & \underline{a}_{j+1} & \ldots \\
i_{2} & \cdots & i_{j-1}
\end{array}\right] .
$$

TheOrem 2.1. Assume that the uniformity assumption

$$
\delta_{t: n}=\max _{1 \leq i \leq n} G_{t i: n}^{\star}\left(x_{t}\right) \rightarrow 0, \quad t=1,2, \text { as } n \rightarrow \infty,
$$

is satisfied. Then the d.f. $\Phi_{k, k^{\prime}: n}^{\star}(\underline{x})$ converges weakly to a nondegenerate d.f. $\Phi_{k, k^{\prime}}(\underline{x})$ if, and only if, for all $\underline{x}$ for which $\Phi_{k, .}\left(x_{1}\right)$ and $\Phi_{., k^{\prime}}\left(x_{2}\right)$ are positive, the limits

$$
\lim _{n \rightarrow \infty} \sum_{i=1}^{n} G_{t i: n}^{\star}\left(x_{t}\right)=h_{t}\left(x_{t}\right), \quad t=1,2,
$$

and

$$
\lim _{n \rightarrow \infty} \sum_{i=1}^{n} G_{i: n}^{\star}(\underline{x})=h(\underline{x})
$$


are finite, and the function

$$
\begin{aligned}
\Phi_{k, k^{\prime}}(\underline{x})= & \sum_{t=0}^{k-1} \sum_{s=0}^{k^{\prime}-1} \sum_{r=0}^{t \wedge s} \frac{1}{(t-r) ! r !(s-r) !}\left(h_{1}\left(x_{1}\right)-h(\underline{x})\right)^{t-r} h^{r}(\underline{x}) \\
& \times\left(h_{2}\left(x_{2}\right)-h(\underline{x})\right)^{s-r} \exp \left(-h_{1}\left(x_{1}\right)-h_{2}\left(x_{2}\right)+h(\underline{x})\right)
\end{aligned}
$$

is a nondegenerate d.f. The actual limit d.f. of $\underline{Z}_{k, k^{\prime}: n}^{\star}$ is the one given in (2.6).

REMARK 2.3. The conditions of Theorem 2.1 show that the d.f. $\Phi_{k, k^{\prime}: n}^{\star}(\underline{x})$ converges weakly to the nondegenerate d.f. $\Phi_{k, k^{\prime}}(\underline{x})$ (which is defined by (2.6)), for all fixed values $k$ and $k^{\prime}$, if there exist at least two fixed integers $k_{0}$ and $k_{0}^{\prime}$ for which the d.f. $\Phi_{k_{0}, k_{0}^{\prime}: n}^{\star}(\underline{x})$ converges weakly to the nondegenerate d.f. $\Phi_{k_{0}, k_{0}^{\prime}}(\underline{x})$. Moreover, the different types of the functions $h_{1}\left(x_{1}\right)$ and $h_{2}\left(x_{2}\right)$ can be determined from Theorem 1.1. For the function $h(\underline{x})$, it is easy to show that for all $\underline{x}$ for which $\Phi_{k, .}\left(x_{1}\right)>0$ and $\Phi_{., k^{\prime}}\left(x_{2}\right)>0$, we have either $0 \leq h(\underline{x})<h_{1}\left(x_{1}\right) \wedge h_{2}\left(x_{2}\right)<\infty$ or $h(\underline{x}) \equiv h_{1}\left(x_{1}\right) \wedge h_{2}\left(x_{2}\right) \equiv 0$.

Proof of Theorem 2.1. Since the proof is somewhat lengthy, we split it up into several steps, some of which are of independent interest.

STEP 1 . For any $0 \leq y_{i}<1 / 2, i=1,2, \ldots$,

$$
\exp \left(-\left(1+\max _{1 \leq j \leq n} y_{j}\right) \sum_{i=1}^{n} y_{i}\right) \leq \prod_{i=1}^{n}\left(1-y_{i}\right) \leq \exp \left(-\sum_{i=1}^{n} y_{i}\right) .
$$

The right-hand inequality remains to hold for all $0 \leq y_{i} \leq 1$.

Proof. The right-hand inequality follows immediately from the trivial inequality $1-y \leq e^{-y}$, for all $y$. The left-hand inequality can be proved by using the inequality $\ln (1-y) \geq-y-y^{2}$ whenever $0 \leq y<1 / 2$ (which is a consequence of the Taylor expansion). Therefore, for all $0 \leq y_{i}<1 / 2$, we have

$$
\begin{aligned}
\ln \prod_{i=1}^{n}\left(1-y_{i}\right) & =\sum_{i=1}^{n} \ln \left(1-y_{i}\right) \geq-\sum_{i=1}^{n} y_{i}-\sum_{i=1}^{n} y_{i}^{2} \\
& \geq-\sum_{i=1}^{n} y_{i}-\sum_{i=1}^{n} y_{i} \max _{1 \leq j \leq n} y_{j} \geq-\left(1+\max _{1 \leq j \leq n} y_{j}\right) \sum_{i=1}^{n} y_{i} .
\end{aligned}
$$

STEP 2. For each fixed $s$ (independent of $n$ ) and for any subset $\mathcal{S}=$ $\left\{i_{1}, \ldots, i_{s}: 1<i_{1}<\ldots<i_{s}<n\right\} \subseteq \mathcal{N}=\{1, \ldots, n\},(2.4)$ and (2.5), in view of the uniformity assumptions in (2.3), yield

$$
\sum_{j \in \mathcal{N}-\mathcal{S}} G_{t j: n}^{\star}\left(x_{t}\right) \rightarrow h_{t}\left(x_{t}\right), \quad t=1,2, \text { as } n \rightarrow \infty .
$$


Moreover, since

$$
\delta_{n}=\max _{1 \leq j \leq n} G_{j: n}^{\star}(\underline{x}) \leq \delta_{1: n} \wedge \delta_{2: n} \rightarrow 0 \quad \text { as } n \rightarrow \infty
$$

we get

$$
\sum_{j \in \mathcal{N}-\mathcal{S}} G_{j: n}^{\star}(\underline{x}) \rightarrow h(\underline{x}) \quad \text { as } n \rightarrow \infty,
$$

for every fixed $s$ and any subset $\mathcal{S} \subseteq \mathcal{N}$.

STEP 3. Let $\left\{a_{t j: n}\right\}_{j=1}^{j=n}, t=1,2,3$, be sequences such that

$$
\varepsilon_{t: n}=\max _{1 \leq j \leq n} a_{t j: n} \rightarrow 0 \quad \text { as } n \rightarrow \infty
$$

and

$$
\sum_{i=1}^{n} a_{t i: n} \rightarrow a_{t}, \quad t=1,2,3, \text { as } n \rightarrow \infty .
$$

Then, for any fixed $s_{1}, s_{2}$ and $s_{3}$, we get

$$
\sum_{\mathcal{Q}} \prod_{t=1}^{s_{1}} a_{1 i_{t}: n} \prod_{t=s_{1}+1}^{s_{1}+s_{2}} a_{2 i_{t}: n} \prod_{t=s_{1}+s_{2}+1}^{s_{1}+s_{2}+s_{3}} a_{3 i_{t}: n} \rightarrow \frac{a_{1}^{s_{1}}}{s_{1} !} \frac{a_{2}^{s_{2}}}{s_{2} !} \frac{a_{3}^{s_{3}}}{s_{3} !} \quad \text { as } n \rightarrow \infty
$$

where $\underline{a}_{t: n}=\left(a_{t 1: n} \ldots a_{t n: n}\right)^{\prime}, t=1,2,3$, and $\mathcal{Q}$ is the set of permutations $\left(i_{1}, \ldots, i_{s_{1}+s_{2}+s_{3}}\right)$ of $\left(1, \ldots, s_{1}+s_{2}+s_{3}\right)$ such that $i_{1}<\ldots<i_{s_{1}}, i_{s_{1}+1}<$ $\ldots<i_{s_{1}+s_{2}}$ and $i_{s_{1}+s_{2}+1}<\ldots<i_{s_{1}+s_{2}+s_{3}}$.

Proof. In view of (2.8),

$$
\left(\sum_{j=1}^{n} a_{1 j: n}\right)^{s_{1}}\left(\sum_{j=1}^{n} a_{2 j: n}\right)^{s_{2}}\left(\sum_{j=1}^{n} a_{3 j: n}\right)^{s_{3}} \rightarrow a_{1}^{s_{1}} a_{2}^{s_{2}} a_{3}^{s_{3}}
$$

which is equivalent to

$$
\sum_{\substack{1 \leq i_{1}, i_{2}, \ldots, i_{s_{1}}, i_{s_{1}+1}, \ldots, i_{s_{1}+s_{2}}, \ldots, i_{s_{1}+s_{2}+s_{3}} \leq n}} \prod_{t \in \mathcal{C}_{1}} a_{1 i_{t}: n} \prod_{t \in \mathcal{C}_{2}} a_{2 i_{t}: n} \prod_{t \in \mathcal{C}_{3}} a_{3 i_{t}: n} \rightarrow a_{1}^{s_{1}} a_{2}^{s_{2}} a_{3}^{s_{3}} \quad \text { as } n \rightarrow \infty
$$

where $\mathcal{C}_{1}=\left\{1, \ldots, s_{1}\right\}, \mathcal{C}_{2}=\left\{s_{1}+1, \ldots, s_{1}+s_{2}\right\}$ and $\mathcal{C}_{3}=\left\{s_{1}+s_{2}+1\right.$, $\left.\ldots, s_{1}+s_{2}+s_{3}\right\}$, or is equivalent to

$$
\left(\sum_{\mathcal{C}}+\sum_{\mathcal{C}^{\prime}}\right) \prod_{t \in \mathcal{C}_{1}} a_{1 i_{t}: n} \prod_{t \in \mathcal{C}_{2}} a_{2 i_{t}: n} \prod_{t \in \mathcal{C}_{3}} a_{3 i_{t}: n} \rightarrow a_{1}^{s_{1}} a_{2}^{s_{2}} a_{3}^{s_{3}} \quad \text { as } n \rightarrow \infty,
$$

where $\sum_{\mathcal{C}}$ signifies summation over those subscripts $\left(i_{1}, \ldots, i_{s_{1}+s_{2}+s_{3}}\right)$ for which all the subscripts related to each $\mathcal{C}_{j}, j=1,2,3$, are different, i.e., $\mathcal{C}=\left\{1 \leq i_{1} \neq \ldots \neq i_{s_{1}} \leq n ; 1 \leq i_{s_{1}+1} \neq \ldots \neq i_{s_{1}+s_{2}} \leq n ; 1 \leq i_{s_{1}+s_{2}+1} \neq\right.$ $\left.\ldots \neq i_{s_{1}+s_{2}+s_{3}} \leq n\right\}$ and $\mathcal{C}^{\prime}$ is the complement of $\mathcal{C}$. Clearly, 


$$
\begin{aligned}
\sum_{\mathcal{C}^{\prime}} \prod_{t \in \mathcal{C}_{1}} a_{1 i_{t}: n} \prod_{t \in \mathcal{C}_{2}} a_{2 i_{t}: n} \prod_{t \in \mathcal{C}_{3}} a_{3 i_{t}: n} & \\
& \leq\left(\sum_{\mathcal{C}_{1}^{\prime}}+\sum_{\mathcal{C}_{2}^{\prime}}+\sum_{\mathcal{C}_{3}^{\prime}}\right) \prod_{t \in \mathcal{C}_{1}} a_{1 i_{t}: n} \prod_{t \in \mathcal{C}_{2}} a_{2 i_{t}: n} \prod_{t \in \mathcal{C}_{3}} a_{3 i_{t}: n}
\end{aligned}
$$

where $\sum_{\mathcal{C}_{j}^{\prime}}$ signifies summation over those subscripts $\left(i_{1}, \ldots, i_{s_{1}+s_{2}+s_{3}}\right)$ for which at least two of the subscripts related to $\mathcal{C}_{j}$ are equal, $j=1,2,3$ (e.g., if $j=2$ (say) then $\sum_{\mathcal{C}_{2}^{\prime}}$ signifies summation over those $\left(i_{1}, \ldots, i_{s_{1}+s_{2}+s_{3}}\right)$ for which at least two of $1 \leq i_{s_{1}+1}, \ldots, i_{s_{1}+s_{2}} \leq n$ are equal). On the other hand, for each $j=1,2,3$, we can write

$$
\sum_{\mathcal{C}_{j}^{\prime}} \prod_{t \in \mathcal{C}_{1}} a_{1 i_{t}: n} \prod_{t \in \mathcal{C}_{2}} a_{2 i_{t}: n} \prod_{t \in \mathcal{C}_{3}} a_{3 i_{t}: n}=\sum_{l=2}^{s_{j}} \sum_{\mathcal{C}_{j l}^{\prime}} \prod_{t \in \mathcal{C}_{1}} a_{1 i_{t}: n} \prod_{t \in \mathcal{C}_{2}} a_{2 i_{t}: n} \prod_{t \in \mathcal{C}_{3}} a_{3 i_{t}: n}
$$

where $\sum_{\mathcal{C}_{j l}^{\prime}}, l=2, \ldots, s_{j}$, signifies summation over those subscripts $\left(i_{1}, \ldots, i_{s_{1}+s_{2}+s_{3}}\right)$ for which $l$ subscripts related to $\mathcal{C}_{j}$ are equal, $l=2, \ldots, s_{j}$, $j=1,2,3$, (e.g., if $j=2$ (say) then $\sum_{\mathcal{C}_{2 l}^{\prime}}$ signifies summation over those $\left(i_{1}, \ldots, i_{s_{1}+s_{2}+s_{3}}\right)$ for which $l$ of $1 \leq i_{s_{1}+1}, \ldots, i_{s_{1}+s_{2}} \leq n$ are equal). However, in view of (2.8), for each $l=2, \ldots, s_{j}$ and $j=1,2,3$, it is easy to verify that, as $n \rightarrow \infty$,

$$
\begin{aligned}
& \sum_{\mathcal{C}_{1 l}^{\prime}} \prod_{t \in \mathcal{C}_{1}} a_{1 i_{t}: n} \prod_{t \in \mathcal{C}_{2}} a_{2 i_{t}: n} \prod_{t \in \mathcal{C}_{3}} a_{3 i_{t}: n} \\
& \quad \leq \varepsilon_{1: n}^{l-1} \sum_{1 \leq i_{1}, \ldots, i_{s_{1}+s_{2}+s_{3}-l+1} \leq n} \prod_{t \in \mathcal{C}_{11}} a_{1 i_{t}: n} \prod_{t \in \mathcal{C}_{21}} a_{2 i_{t}: n} \prod_{t \in \mathcal{C}_{31}} a_{3 i_{t}: n} \rightarrow 0 \\
& \sum_{\mathcal{C}_{2 l}^{\prime}} \prod_{t \in \mathcal{C}_{1}} a_{1 i_{t}: n} \prod_{t \in \mathcal{C}_{2}} a_{2 i_{t}: n} \prod_{t \in \mathcal{C}_{3}} a_{3 i_{t}: n} \\
& \quad \leq \varepsilon_{2: n}^{l-1} \sum_{1 \leq i_{1}, \ldots, i_{s_{1}+s_{2}+s_{3}-l+1} \leq n} \prod_{t \in \mathcal{C}_{1}} a_{1 i_{t}: n} \prod_{t \in \mathcal{C}_{22}} a_{2 i_{t}: n} \prod_{t \in \mathcal{C}_{32}} a_{3 i_{t}: n} \rightarrow 0
\end{aligned}
$$

and

$$
\begin{aligned}
\sum_{\mathcal{C}_{3 l}^{\prime}} & \prod_{t \in \mathcal{C}_{1}} a_{1 i_{t}: n} \prod_{t \in \mathcal{C}_{2}} a_{2 i_{t}: n} \prod_{t \in \mathcal{C}_{3}} a_{3 i_{t}: n} \\
& \leq \varepsilon_{3: n}^{l-1} \sum_{1 \leq i_{1}, \ldots, i_{s_{1}+s_{2}+s_{3}-l+1} \leq n} \prod_{t \in \mathcal{C}_{1}} a_{1 i_{t}: n} \prod_{t \in \mathcal{C}_{2}} a_{2 i_{t}: n} \prod_{t \in \mathcal{C}_{33}} a_{3 i_{t}: n} \rightarrow 0
\end{aligned}
$$

where $\mathcal{C}_{11}=\left\{1, \ldots, s_{1}-l+1\right\}, \mathcal{C}_{21}=\left\{s_{1}-l+2, \ldots, s_{1}+s_{2}-l+1\right\}$, $\mathcal{C}_{31}=\left\{s_{1}+s_{2}-l+2, \ldots, s_{1}+s_{2}+s_{3}-l+1\right\}, \mathcal{C}_{22}=\left\{s_{1}+1, \ldots, s_{1}+s_{2}-l+1\right\}$, $\mathcal{C}_{32}=\left\{s_{1}+s_{2}-l+2, \ldots, s_{1}+s_{2}+s_{3}-l+1\right\}$ and $\mathcal{C}_{33}=\left\{s_{1}+s_{2}+1, \ldots\right.$ 
$\left.\ldots, s_{1}+s_{2}+s_{3}-l+1\right\}$, from which we deduce that

$$
\sum_{\mathcal{C}^{\prime}} \prod_{t \in \mathcal{C}_{1}} a_{1 i_{t}: n} \prod_{t \in \mathcal{C}_{2}} a_{2 i_{t}: n} \prod_{t \in \mathcal{C}_{3}} a_{3 i_{t}: n} \rightarrow 0 \quad \text { as } n \rightarrow \infty .
$$

On the other hand, we have

$$
\begin{aligned}
\sum_{\mathcal{C}} \prod_{t \in \mathcal{C}_{1}} a_{1 i_{t}: n} \prod_{t \in \mathcal{C}_{2}} a_{2 i_{t}: n} \prod_{t \in \mathcal{C}_{3}} a_{3 i_{t}: n} \\
\quad=s_{1} ! s_{2} ! s_{3} ! \sum_{\mathcal{Q}} \prod_{t \in \mathcal{C}_{1}} a_{1 i_{t}: n} \prod_{t \in \mathcal{C}_{2}} a_{2 i_{t}: n} \prod_{t \in \mathcal{C}_{3}} a_{3 i_{t}: n} .
\end{aligned}
$$

Hence the result.

STEP 4. Let $n$ be sufficiently large such that $G_{t i: n}^{\star}\left(x_{t}\right)<1 / 2, t=1,2$ (consequently, $G_{i: n}^{\star}(\underline{x})<1 / 2$ ). Then

$$
\sum_{t=0}^{k-1} \sum_{s=0}^{k^{\prime}-1} \sum_{r=0 \vee(t+s-n)}^{t \wedge s} \sum_{\mathcal{P}_{r, s, t}} \prod_{j=1}^{t-r}\left(G_{1 i_{j}: n}^{\star}\left(x_{1}\right)-G_{i_{j}: n}^{\star}(\underline{x})\right)
$$

$$
\begin{aligned}
& \times \prod_{j=t-r+1}^{t} G_{i_{j}: n}^{\star}(\underline{x}) \prod_{j=t+1}^{t+s-r}\left(G_{2 i_{j}: n}^{\star}\left(x_{2}\right)-G_{i_{j}: n}^{\star}(\underline{x})\right) \\
& \times e^{-\left(1+\delta_{1: n}+\delta_{2: n}\right)} \sum_{j=t+s-r+1}^{n}\left(G_{1 i_{j}: n}^{\star}\left(x_{1}\right)+G_{2 i_{j}: n}^{\star}\left(x_{2}\right)-G_{i_{j}: n}^{\star}(\underline{x})\right)
\end{aligned}
$$

$$
\leq \Phi_{k, k^{\prime}: n}^{\star}(\underline{x})
$$$$
\leq \sum_{t=0}^{k-1} \sum_{s=0}^{k^{\prime}-1} \sum_{r=0 \vee(t+s-n)}^{t \wedge s} \sum_{\mathcal{P}_{r, s, t}} \prod_{j=1}^{t-r}\left(G_{1 i_{j}: n}^{\star}\left(x_{1}\right)-G_{i_{j}: n}^{\star}(\underline{x})\right) \prod_{j=t-r+1}^{t} G_{i_{j}: n}^{\star}(\underline{x})
$$

$$
\times \prod_{j=t+1}^{t+s-r}\left(G_{2 i_{j}: n}^{\star}\left(x_{2}\right)-G_{i_{j}: n}^{\star}(\underline{x})\right) e^{-\sum_{j=t+s-r+1}^{n}\left(G_{1 i_{j}: n}^{\star}\left(x_{1}\right)+G_{2 i_{j}: n}^{\star}\left(x_{2}\right)-G_{i_{j}: n}^{\star}(\underline{x})\right)} .
$$

Proof. Upon replacing the functions $\Phi_{k, k^{\prime}: n}(\underline{x}), G_{1 j}\left(x_{1}\right), G_{2 j}\left(x_{2}\right)$ and $G_{j}(\underline{x}), j=1, \ldots, n$, in $(2.1)$ respectively by $\Phi_{k, k^{\prime}: n}^{\star}(\underline{x}), G_{1 j: n}^{\star}\left(x_{1}\right), G_{2 j: n}^{\star}\left(x_{2}\right)$ and $G_{j: n}^{\star}(\underline{x}), j=1, \ldots, n$, the proof follows immediately by applying inequality (2.7) to $(2.1)$ (note that $\max _{t+s-r+1 \leq j \leq n}\left(G_{1 i_{j}: n}^{\star}\left(x_{1}\right)+G_{2 i_{j}: n}^{\star}\left(x_{2}\right)-\right.$ $\left.\left.G_{i_{j}: n}^{\star}(\underline{x})\right) \leq \delta_{1: n}+\delta_{2: n}\right)$.

We now conclude the first part of Theorem 2.1 by taking the limit of (2.9), as $n \rightarrow \infty$, as follows. Apply Step 3 with $\underline{a}_{1: n}=\left(G_{11: n}^{\star}\left(x_{1}\right)-\right.$ $\left.G_{1: n}^{\star}(\underline{x}), G_{12: n}^{\star}\left(x_{1}\right)-G_{2: n}^{\star}(\underline{x}), \ldots, G_{1 n: n}^{\star}\left(x_{1}\right)-G_{n: n}^{\star}(\underline{x})\right)^{\prime}, \underline{a}_{2: n}=\left(G_{1: n}^{\star}(\underline{x}), \ldots\right.$ $\left.\ldots, G_{n: n}^{\star}(\underline{x})\right)^{\prime}, \underline{a}_{3: n}=\left(G_{21: n}^{\star}\left(x_{2}\right)-G_{1: n}^{\star}(\underline{x}), G_{22: n}^{\star}\left(x_{2}\right)-G_{2: n}^{\star}(\underline{x}), \ldots, G_{2 n: n}^{\star}\left(x_{2}\right)\right.$ $\left.-G_{n: n}^{\star}(\underline{x})\right)^{\prime}, s_{1}=t-r, s_{2}=r$ and $s_{3}=s-r$. On the other hand, by applying Step 2 with $\mathcal{S}=\left\{i_{1}, \ldots, i_{s_{1}+s_{2}+s_{3}}\right\}$, the sum in the exponent of $e$ in (2.9) tends to $h_{1}\left(x_{1}\right)+h_{2}\left(x_{2}\right)-h(\underline{x})$. This completes the proof. 
We now turn to the proof of the converse of the theorem. The idea of the proof is based heavily on the proof of Theorem 5.3.1 of Galambos (1987) (the converse part of Theorem 5.3.1). Namely, we assume that the uniformity assumptions in (2.3) are satisfied. Furthermore, assume the d.f. of $Z_{k, k^{\prime}: n}^{\star}$ converges weakly to a limit d.f. $\Phi_{k, k^{\prime}}(\underline{x})$, given by (2.6). Let $\underline{x}$ be such that $\Phi_{k, .}\left(x_{1}\right)>0$ and $\Phi_{., k^{\prime}}\left(x_{2}\right)>0$. We shall prove the validity of $(2.4)$, for $t=1,2$, and (2.5). The proof will be accomplished in the following two steps.

STEP 5. For any fixed $h_{1}, h_{2}$ and all values of $h$ for which $0<h<$ $h_{1} \wedge h_{2}<\infty$, the function

$\widetilde{\Phi}_{k, k^{\prime}}\left(h_{1}, h_{2}, h\right)$

$=\sum_{t=0}^{k-1} \sum_{s=0}^{k^{\prime}-1} \sum_{r=0}^{t \wedge s} \frac{1}{(t-r) ! r !(s-r) !}\left(h_{1}-h\right)^{t-r} h^{r}\left(h_{2}-h\right)^{s-r} \exp \left(-h_{1}-h_{2}+h\right)$

is uniquely determined by $h$.

Proof. The proof immediately follows if we observe that

$$
\begin{aligned}
\exp \left(h_{1}+h_{2}-h\right) \frac{\partial \widetilde{\Phi}_{k, k^{\prime}}\left(h_{1}, h_{2}, h\right)}{\partial h} & -\sum_{j=0}^{k^{\prime}-1} \sum_{i=1}^{k-1} \sum_{r=0}^{(i-1) \wedge j} \frac{\left(h_{1}-h\right)^{i-r-1} h^{r}\left(h_{2}-h\right)^{j-r}}{(i-r-1) ! r !(j-r) !} \\
& -\sum_{j=1}^{k^{\prime}-1} \sum_{i=0}^{k-1} \sum_{r=0}^{i \wedge(j-1)} \frac{\left(h_{1}-h\right)^{i-r} h^{r}\left(h_{2}-h\right)^{j-r-1}}{(i-r) ! r !(j-r-1) !} \\
& +\sum_{j=1}^{k^{\prime}-1} \sum_{i=1}^{k-1} \sum_{r=1}^{i \wedge j} \frac{\left(h_{1}-h\right)^{i-r} h^{r-1}\left(h_{2}-h\right)^{j-r}}{(i-r) !(r-1) !(j-r) !} \\
& +\sum_{j=0}^{k^{\prime}-1} \sum_{i=0}^{k-1} \sum_{r=0}^{i \wedge j} \frac{\left(h_{1}-h\right)^{i-r} h^{r}\left(h_{2}-h\right)^{j-r}}{(i-r) ! r !(j-r) !},
\end{aligned}
$$

which after routine calculations yields

$$
\begin{aligned}
& \frac{\partial \widetilde{\Phi}_{k, k^{\prime}}\left(h_{1}, h_{2}, h\right)}{\partial h} \\
& \quad=\sum_{r=0}^{(k-1) \wedge\left(k^{\prime}-1\right)} \frac{\left(h_{1}-h\right)^{k-r-1} h^{r}\left(h_{2}-h\right)^{k^{\prime}-r-1}}{(k-r-1) ! r !\left(k^{\prime}-r-1\right) !} \exp \left(-h_{1}-h_{2}+h\right)>0,
\end{aligned}
$$

when $h_{1}$ and $h_{2}$ are fixed and $0<h<h_{1} \wedge h_{2}<\infty$, i.e., the function $\widetilde{\Phi}_{k, k^{\prime}}\left(h_{1}, h_{2}, h\right)$ is strictly increasing in $h$ when $h_{1}$ and $h_{2}$ are fixed (see Lemma 2.2 of Barakat, 1999, and Barakat, 1997). Hence the result. 
STEP 6. Since (2.6) implies (2.4) for $t=1,2$ (by letting respectively $x_{1} \rightarrow \infty, x_{2} \rightarrow \infty$ and by applying Theorem 1.1), the elementary inequalities $G_{j: n}^{\star}(\underline{x}) \leq G_{1 j: n}^{\star}\left(x_{1}\right) \wedge G_{2 j: n}^{\star}\left(x_{2}\right), j=1, \ldots, n$, yield that $\left\{\sum_{j=1}^{n} G_{j: n}^{\star}(\underline{x})\right\}$ is bounded. Therefore, we can select a subsequence $\left\{n^{\prime}\right\}$ of $\{n\}$ for which (2.5) holds. Let us repeat the first part of the proof (Steps 1-4) for this subsequence. We find that the limit $\Phi_{k, k^{\prime}}(\underline{x})$ of $\Phi_{k, k^{\prime}: n^{\prime}}^{\star}(\underline{x})$ satisfies $(2.6)$ where the limit in (2.5) may depend on the actual subsequence $\left\{n^{\prime}\right\}$. However, in view of the result of Step 5, we conclude from the representation (2.6) that (2.5) cannot depend on $n^{\prime}$. This completes the proof.

Corollary 2.1. Let (2.3)-(2.5) be satisfied with $a_{1 n}=a_{2 n}$ and $b_{1 n}=$ $b_{2 n}$. Then $\omega_{1} \equiv \omega_{2} \leq \infty$, where $\omega_{1}=\sup \left\{x_{1}: \Phi_{k, .}\left(x_{1}\right)<1\right\}$ and $\omega_{2}=$ $\sup \left\{x_{2}: \Phi_{., k^{\prime}}\left(x_{2}\right)<1\right\}$.

Proof. We first note that Theorem A.3 of Balkema et al. (1993) is closely related to the special case $k=k^{\prime}=1$ of Theorem 2.1. Therefore, together with Remark 2.3 it implies the conclusion of the present corollary.

The following result is an immediate consequence of Theorem 2.1.

Corollary 2.2. Assume that $\underline{Z}_{k, k^{\prime}: n}^{\star}$ has a nondegenerate asymptotic distribution $\Phi_{k, k^{\prime}}(\underline{x})$. Then the components of $\underline{Z}_{k, k^{\prime}: n}^{\star}$ are asymptotically independent if, and only if, the limit in (2.5) is identically zero i.e., $h(\underline{x})=0$.

We are now in a position to obtain a general condition under which the components of the vector $\underline{Z}_{k, k^{\prime}: n}^{\star}$ will be asymptotically independent. The following remark will be needed.

REMARK 2.4. Let $\bar{F}_{t: n}\left(x_{t}\right)=n^{-1} \sum_{j=1}^{n} F_{t j: n}\left(x_{t}\right), t=1,2$, and $\bar{F}_{n}(\underline{x})=$ $n^{-1} \sum_{j=1}^{n} F_{j}(\underline{x})$. Then, keeping the notations of Section 1, (2.4) and (2.5) may be written respectively as $n \bar{G}_{t: n}^{\star}\left(x_{t}\right)=n\left(1-\bar{F}_{t: n}^{\star}\left(x_{t}\right)\right) \rightarrow h_{t}\left(x_{t}\right), t=$ 1,2 , as $n \rightarrow \infty$ and $n \bar{G}_{n}^{\star}(\underline{x}) \rightarrow h(\underline{x})$ as $n \rightarrow \infty$, where $\bar{G}_{t: n}^{\star}\left(x_{t}\right), t=1,2$, and $\bar{G}_{n}^{\star}(\underline{x})$ denote respectively the survival functions of $\bar{F}_{t: n}^{\star}\left(x_{t}\right), t=1,2$, and $\bar{F}_{n}^{\star}(\underline{x})$.

TheOREM 2.2. Let $\widehat{x}_{t: n}=\sup \left\{x_{t}: \bar{F}_{t: n}\left(x_{t}\right)<1\right\}, t=1,2$. Let further $\lim _{n \rightarrow \infty} \widehat{x}_{t: n}=\widehat{x}_{t} \leq \infty, t=1,2$. Then, the components of the vector $\underline{Z}_{k, k^{\prime}: n}^{\star}$ are asymptotically independent if, and only if

$$
\lim _{\substack{n \rightarrow \infty \\ \underline{x} \rightarrow \underline{x}}} \frac{\bar{G}_{n}(\underline{x})}{1-\bar{F}_{n}(\underline{x})}=0,
$$

where $\underline{\widehat{x}}=\left(\widehat{x}_{1}, \widehat{x}_{2}\right)$.

Proof. We first observe that, in view of Corollary 2.2, the components of the vector $\underline{Z}_{k, k^{\prime}: n}^{\star}$ are asymptotically independent if, and only if, $h(\underline{x})=0$. Thus, in order to prove the theorem, we have to prove $h(\underline{x})=0$ if, and only 
if, (2.10) holds. On the other hand, in view of the elementary relation

$$
\bar{G}_{n}(\underline{x})=\bar{G}_{1: n}\left(x_{1}\right)+\bar{G}_{2: n}\left(x_{2}\right)-\left(1-\bar{F}_{n}(\underline{x})\right),
$$

(2.10) is equivalent to

$$
\lim _{\substack{n \rightarrow \infty \\ \underline{x \rightarrow \underline{x}}}} \frac{\bar{G}_{1: n}\left(x_{1}\right)+\bar{G}_{2: n}\left(x_{2}\right)}{\bar{G}_{1: n}\left(x_{1}\right)+\bar{G}_{2: n}\left(x_{2}\right)-\bar{G}_{n}(\underline{x})}=1 .
$$

Suppose now that (2.12) holds. Appealing to (2.4), (2.5) and Remark 2.4 , we can easily get

$$
\frac{h_{1}\left(x_{1}\right)+h_{2}\left(x_{2}\right)}{h_{1}\left(x_{1}\right)+h_{2}\left(x_{2}\right)-h(\underline{x})}=1
$$

for all $\underline{x}$ such that $h_{1}\left(x_{1}\right), h_{2}\left(x_{2}\right)<\infty$, i.e., for all $\underline{x}$ such that $\Phi_{k, .}\left(x_{1}\right)$, $\Phi_{., k^{\prime}}\left(x_{2}\right)>0$ (note that, in view of Remark 2.4, $\underline{a}_{n} \underline{x}+\underline{b}_{n} \rightarrow \underline{\widehat{x}}$ when $\left.\Phi_{k, .}\left(x_{1}\right), \Phi_{k, k^{\prime}}\left(x_{2}\right)>0\right)$, which yields $h_{1}\left(x_{1}\right)+h_{2}\left(x_{2}\right)-h(\underline{x})=h_{1}\left(x_{1}\right)+$ $h_{2}\left(x_{2}\right)$, i.e., $h(\underline{x})=0$. Conversely, suppose that $h(\underline{x})=0$ and (2.10) does not hold. Then there exist $\varepsilon>0$ and sequences $\left\{\underline{v}_{m}\right\}=\left\{\left(v_{1 m}, v_{2 m}\right)\right\},\left\{n_{m}\right\}$ such that $\underline{v}_{m} \rightarrow \underline{\widehat{x}}$ and $n_{m} \rightarrow \infty$ as $m \rightarrow \infty$, and

$$
\bar{G}_{1: n_{m}}\left(v_{1 m}\right)+\bar{G}_{2: n_{m}}\left(v_{2 m}\right) \geq \bar{G}_{n_{m}}\left(\underline{v}_{m}\right) \geq \varepsilon\left(1-\bar{F}_{n_{m}}\left(\underline{v}_{m}\right)\right) \text {. }
$$

Now choose a subsequence $\left\{n_{l}^{\star}\right\}=\left\{n_{m_{l}}\right\}$ of $\left\{n_{m}\right\}$ so that

$$
\begin{aligned}
\frac{h_{1}\left(x_{1}\right)+h_{2}\left(x_{2}\right)}{n_{l}^{\star}+1} & \leq \bar{G}_{1: n_{l}^{\star}}\left(v_{1 l}^{\star}\right)+\bar{G}_{2: n_{l}^{\star}}\left(v_{2 l}^{\star}\right)-2 \bar{G}_{n_{l}^{\star}}\left(\underline{v}_{l}^{\star}\right) \\
& \leq \bar{G}_{1: n_{l}^{\star}}\left(v_{1 l}^{\star}\right)+\bar{G}_{2: n_{l}^{\star}}\left(v_{2 l}^{\star}\right) \leq \frac{h_{1}\left(x_{1}\right)+h_{2}\left(x_{2}\right)}{n_{l}^{\star}} .
\end{aligned}
$$

(Note that $n \bar{G}_{n}^{\star}(\underline{x}) \rightarrow 0$. Therefore, $n_{l}^{\star} \bar{G}_{n_{l}^{\star}}^{\star}\left(\underline{v}_{l}^{\star}\right) \rightarrow 0$, where $\underline{v}_{m_{l}}=\underline{v}_{l}^{\star}=$ $\left(v_{1 l}^{\star}, v_{2 l}^{\star}\right)$ and $\left\{\underline{v}_{m_{l}}\right\}$ is a subsequence of $\left\{\underline{v}_{m}\right\}$.) Clearly, we have either

(i) $\bar{F}_{n_{l}^{\star}}^{\star}(\underline{x}) \geq \bar{F}_{n_{l}^{\star}}^{\star}\left(\underline{v}_{l}^{\star}\right)$ for infinitely many $l$, or

(ii) $\bar{F}_{n_{l}^{\star}}^{\star}(\underline{x})<\bar{F}_{n_{l}^{\star}}^{\star}\left(\underline{v}_{l}^{\star}\right)$ for infinitely many $l$.

If (i) holds, then for such $l$,

$$
n_{l}^{\star}\left(1-\bar{F}_{n_{l}^{\star}}^{\star}(\underline{x})\right) \leq n_{l}^{\star}\left(1-\bar{F}_{n_{l}^{\star}}^{\star}\left(\underline{v}_{l}^{\star}\right)\right) .
$$

Clearly, we have

$$
\begin{aligned}
n_{l}^{\star}\left(1-\bar{F}_{n_{l}^{\star}}^{\star}\left(\underline{v}_{l}^{\star}\right)\right)=h_{1}\left(x_{1}\right)+h_{2}\left(x_{2}\right) \\
\quad+n_{l}^{\star}\left(-\frac{h_{1}\left(x_{1}\right)+h_{2}\left(x_{2}\right)}{n_{l}^{\star}}+\bar{G}_{1: n_{l}^{\star}}\left(v_{1 l}^{\star}\right)+\bar{G}_{2: n_{l}^{\star}}\left(v_{2 l}^{\star}\right)-\bar{G}_{n_{l}^{\star}}\left(\underline{v}_{l}^{\star}\right)\right) \\
\leq h_{1}\left(x_{1}\right)+h_{2}\left(x_{2}\right) \\
\quad+n_{l}^{\star}\left(-\frac{h_{1}\left(x_{1}\right)+h_{2}\left(x_{2}\right)}{n_{l}^{\star}}+\frac{h_{1}\left(x_{1}\right)+h_{2}\left(x_{2}\right)}{n_{l}^{\star}}-\varepsilon\left(1-\bar{F}_{n_{l}^{\star}}^{\star}\left(\underline{v}_{l}^{\star}\right)\right)\right),
\end{aligned}
$$


which yields

$$
(1+\varepsilon) n_{l}^{\star}\left(1-\bar{F}_{n_{l}^{\star}}^{\star}\left(\underline{v}_{l}^{\star}\right)\right) \leq h_{1}\left(x_{1}\right)+h_{2}\left(x_{2}\right) .
$$

It follows that

$$
\liminf _{l \rightarrow \infty} n_{l}^{\star}\left(1-\bar{F}_{n_{l}^{\star}}^{\star}\left(\underline{v}_{l}^{\star}\right)\right)<h_{1}\left(x_{1}\right)+h_{2}\left(x_{2}\right),
$$

and hence by (2.15),

$$
\liminf _{l \rightarrow \infty} n_{l}^{\star}\left(1-\bar{F}_{n_{l}^{\star}}^{\star}(\underline{x})\right)<h_{1}\left(x_{1}\right)+h_{2}\left(x_{2}\right),
$$

which contradicts the fact that $n\left(1-\bar{F}_{n}^{\star}(\underline{x})\right)=n \bar{G}_{1: n}^{\star}\left(x_{1}\right)+n \bar{G}_{2: n}^{\star}\left(x_{2}\right)-$ $n \bar{G}_{n}^{\star}(\underline{x}) \rightarrow h_{1}\left(x_{1}\right)+h_{2}\left(x_{2}\right)$ as $n \rightarrow \infty$ (note that $\left.n \bar{G}_{n}^{\star}(\underline{x}) \rightarrow 0\right)$.

If (ii) holds, then for such $\ell$,

$$
n_{\ell}^{\star}\left(1-\bar{F}_{n_{\ell}^{\star}}^{\star}(\underline{x})\right)>n_{\ell}^{\star}\left(1-\bar{F}_{n_{\ell}^{\star}}^{\star}\left(\underline{v}_{\ell}^{\star}\right)\right) .
$$

Clearly, we have (in view of (2.13) and (2.14))

$$
\begin{aligned}
n_{l}^{\star} & \left(1-\bar{F}_{n_{l}^{\star}}^{\star}\left(\underline{v}_{l}^{\star}\right)\right)=h_{1}\left(x_{1}\right)+h_{2}\left(x_{2}\right) \\
& +n_{l}^{\star}\left(-\frac{h_{1}\left(x_{1}\right)+h_{2}\left(x_{2}\right)}{n_{l}^{\star}}+\left(\bar{G}_{1: n_{l}^{\star}}\left(v_{1 l}^{\star}\right)+\bar{G}_{2: n_{l}^{\star}}\left(v_{2 l}^{\star}\right)-2 \bar{G}_{n_{l}^{\star}}\left(\underline{v}_{l}^{\star}\right)\right)+\bar{G}_{n_{l}^{\star}}\left(\underline{v}_{l}^{\star}\right)\right) \\
\geq & h_{1}\left(x_{1}\right)+h_{2}\left(x_{2}\right) \\
& +n_{l}^{\star}\left(-\frac{h_{1}\left(x_{1}\right)+h_{2}\left(x_{2}\right)}{n_{l}^{\star}}+\frac{h_{1}\left(x_{1}\right)+h_{2}\left(x_{2}\right)}{n_{l}^{\star}+1}+\varepsilon\left(1-\bar{F}_{n_{l}^{\star}}^{\star}\left(\underline{v}_{l}^{\star}\right)\right)\right) \\
= & h_{1}\left(x_{1}\right)+h_{2}\left(x_{2}\right)+\varepsilon n_{l}^{\star}\left(1-\bar{F}_{n_{l}^{\star}}^{\star}\left(\underline{v}_{l}^{\star}\right)\right)-\frac{h_{1}\left(x_{1}\right)+h_{2}\left(x_{2}\right)}{n_{l}^{\star}+1},
\end{aligned}
$$

which yields

$$
(1-\varepsilon) n_{l}^{\star}\left(1-\bar{F}_{n_{l}^{\star}}^{\star}\left(\underline{v}_{l}^{\star}\right)\right) \geq h_{1}\left(x_{1}\right)+h_{2}\left(x_{2}\right)-\frac{h_{1}\left(x_{1}\right)+h_{2}\left(x_{2}\right)}{n_{l}^{\star}+1} .
$$

Since clearly $n_{l}^{\star} \rightarrow \infty$, it follows that (since $0<h_{1}\left(x_{1}\right)+h_{2}\left(x_{2}\right)<\infty$ by assumption)

$$
\limsup _{l \rightarrow \infty} n_{l}^{\star}\left(1-\bar{F}_{n_{l}^{\star}}^{\star}\left(\underline{v}_{l}^{\star}\right)\right)>h_{1}\left(x_{1}\right)+h_{2}\left(x_{2}\right),
$$

and hence by $(2.16)$,

$$
\limsup _{l \rightarrow \infty} n_{l}^{\star}\left(1-\bar{F}_{n_{l}^{\star}}^{\star}(\underline{x})\right)>h_{1}\left(x_{1}\right)+h_{2}\left(x_{2}\right),
$$

which contradicts again the fact that $n\left(1-\bar{F}_{n}^{\star}(\underline{x})\right)=n \bar{G}_{1: n}^{\star}\left(x_{1}\right)+n \bar{G}_{2: n}^{\star}\left(x_{2}\right)-$ $n \bar{G}_{n}^{\star}(\underline{x}) \rightarrow h_{1}\left(x_{1}\right)+h_{2}\left(x_{2}\right)$, as $n \rightarrow \infty$. Hence the result.

REMARK 2.5. Theorem 2.2 can be considered as a substantial generalization of Theorem 2.3 of Barakat (1999). On the other hand, Theorem 2.3 
proves the necessity of the Geffroy condition (see Sarhan and Greenberg, 1962) in the case of independent and identically distributed r.v.'s.

We now briefly go over parallel results for $\underline{V}_{k, k^{\prime}: n}$. Keeping the notation of Remark 2.1 and using the elementary relation $\min \left(X_{11}, X_{12}, \ldots, X_{1 n}\right)=$ $-\max \left(-X_{11},-X_{12}, \ldots,-X_{1 n}\right)$, it is easy to write explicitly the d.f. of $\underline{V}_{k, k^{\prime}: n}$ :

$$
\Psi_{k, k^{\prime}: n}(\underline{x})=\Phi_{., k^{\prime}: n}\left(x_{2}\right)-M_{k, k^{\prime}: n}(\underline{x}),
$$

where

$$
\begin{aligned}
M_{k, k^{\prime}: n}(\underline{x})= & P\left(X_{1, k: n} \geq x_{1}, X_{2, n-k^{\prime}+1: n}<x_{2}\right) \\
= & \sum_{t=0}^{k-1} \sum_{s=0}^{k^{\prime}-1} \sum_{r=0 \vee(t+s-n)}^{t \wedge s} \sum_{\mathcal{P}_{r, s, t}} \prod_{j=1}^{t-r} F_{i_{j}}(\underline{x}) \\
& \times \prod_{j=t-r+1}^{t}\left(F_{1 i_{j}}\left(x_{1}\right)-F_{i_{j}}(\underline{x})\right) \prod_{j=t+1}^{t+s-r} G_{i_{j}}(\underline{x}) \\
& \times \prod_{j=t+s-r+1}^{n}\left(1-G_{2 i_{j}}\left(x_{2}\right)+F_{i_{j}}(\underline{x})\right) .
\end{aligned}
$$

Moreover, for any sequence of suitable normalizing constants $\underline{a}_{n}^{\prime}=\left(\alpha_{1 n}, a_{2 n}\right)$ $>\underline{0}$ and $\underline{b}_{n}^{\prime}=\left(\beta_{1 n}, b_{2 n}\right)$, in view of the identity $\sum_{i=1}^{n} G_{i: n}^{\star}(\underline{x})=\sum_{i=1}^{n} G_{2 i: n}^{\star}\left(x_{2}\right)$ $-\sum_{i=1}^{n} F_{1 i: n}^{\star}\left(x_{1}\right)+\sum_{i=1}^{n} F_{i: n}^{\star}(\underline{x})$ we have the following theorem.

Theorem 2.3. Assume that the uniformity assumptions

$$
\delta_{1: n}^{\prime}=\max _{1 \leq i \leq n} F_{1 i: n}^{\star}\left(x_{1}\right) \rightarrow 0 \quad \text { and } \quad \delta_{2: n}=\max _{1 \leq i \leq n} G_{2 i: n}^{\star}\left(x_{2}\right) \rightarrow 0
$$

as $n \rightarrow \infty$ are satisfied. Then the d.f. $\Psi_{k, k^{\prime}: n}^{\star}(\underline{x})=\Psi_{k, k^{\prime}: n}^{\star}\left(\underline{a}_{n}^{\prime} \underline{x}+\underline{b}_{n}^{\prime}\right)$ converges weakly to a nondegenerate d.f. $\Psi_{k, k^{\prime}}(\underline{x})$ if, and only if, for all $\underline{x}$ for which the two marginals $\Psi_{k, .}\left(x_{1}\right)$ and $\Psi_{., k^{\prime}}\left(x_{2}\right)$ of $\Psi_{k, k^{\prime}}(\underline{x})$ are positive, the limits

$$
\lim _{n \rightarrow \infty} \sum_{i=1}^{n} F_{1 i: n}^{\star}\left(x_{1}\right)=\ell_{1}\left(x_{1}\right), \quad \lim _{n \rightarrow \infty} \sum_{i=1}^{n} G_{2 i: n}^{\star}\left(x_{2}\right)=h_{2}\left(x_{2}\right)
$$

are finite, and moreover at least one of the limits

$$
\lim _{n \rightarrow \infty} \sum_{i=1}^{n} G_{i: n}^{\star}(\underline{x})=h^{\prime}(\underline{x}), \quad \lim _{n \rightarrow \infty} \sum_{i=1}^{n} F_{i: n}^{\star}(\underline{x})=\ell^{\prime}(\underline{x})
$$

is finite. The function $\Psi_{k, k^{\prime}}(\underline{x})=\Phi_{., k^{\prime}}\left(x_{2}\right)-M_{k, k^{\prime}}(\underline{x})$, where

$$
\begin{aligned}
M_{k, k^{\prime}}(\underline{x})= & \sum_{t=0}^{k-1} \sum_{s=0}^{k^{\prime}-1} \sum_{r=0}^{t \wedge s} \frac{1}{(t-r) ! r !(s-r) !} \\
& \times \ell^{\prime t-r}(\underline{x})\left(\ell_{1}\left(x_{1}\right)-\ell^{\prime}(\underline{x})\right)^{r} h^{\prime s-r}(\underline{x}) e^{-h_{2}\left(x_{2}\right)-\ell^{\prime}(\underline{x})},
\end{aligned}
$$


is a nondegenerate d.f. The actual limit d.f. of $\underline{V}_{k, k^{\prime}: n}^{\star}=\left(\underline{V}_{k, k^{\prime}: n}^{\star}-\underline{b}_{n}^{\prime}\right) / \underline{a}_{n}^{\prime}$ is the one given in (2.17). The components of $\underline{V}_{k, k^{\prime}: n}^{\star}$ are asymptotically independent if and only if either $h^{\prime}(\underline{x})=h_{2}\left(x_{2}\right)$ or $\ell^{\prime}(\underline{x})=\ell_{1}\left(x_{1}\right)$.

Finally, we have the following theorem.

THEOREM 2.3. Let $\check{x}_{1: n}=\inf \left\{x_{1}: \bar{F}_{1: n}\left(x_{1}\right)>0\right\}$. Let further $\lim _{n \rightarrow \infty} \check{x}_{1: n}$ $=\check{x}_{1} \geq-\infty$. Then the components of the vector $\underline{V}_{k, k^{\prime}: n}^{\star}$ are asymptotically independent if, and only if,

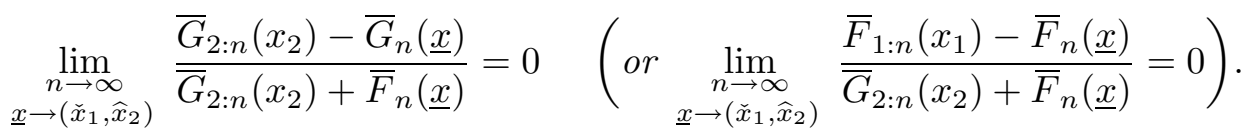

Acknowledgments. The author is grateful to the anonymous referee for his valuable detailed comments and for pointing out several typographical mistakes, which helped considerably in improving the final form of this paper.

\section{References}

N. Balakrishnan (1994), Order statistics from non-identically exponential random variables and some applications, Comput. Statist. Data Anal. 18, 203-225.

A. A. Balkema, L. de Hann and R. L. Karandikar (1993), Asymptotic distribution of the maximum of $n$ independent stochastic processes, J. Appl. Probab. 30, 66-81.

H. M. Barakat (1997), Asymptotic properties of bivariate random extremes, J. Statist. Plann. Inference 61, 203-217.

H. M. Barakat (1999), On the limit behaviour of bivariate extremes, Statistica (Bologna), no. $2,223-237$.

G. Cao and M. West (1997), Computing distributions of order statistics, Comm. Statist. Theory Methods 26, 755-764.

J. Galambos (1987), The Asymptotic Theory of Extreme Order Statistics, 2nd ed., Krieger, Malabar, FL.

L. de Hann and E. Verkade (1985), On extreme value theory in the presence of a trend, technical report, Erasmus Univ.

M. L. Juncosa (1949), The asymptotic behavior of the minimum in a sequence of random variables, Duke Math. J. 16, 609-618.

D. G. Mejzler (1949), On a theorem of B. V. Gnedenko, Sb. Trudov Inst. Mat. Akad. Nauk Ukrain. SSR, no. 12, 31-35 (in Russian).

D. G. Mejzler (1950), On the limit distribution of the maximal term of a variational series, Dopovidi Akad. Nauk. Ukrain. SSR, no. 1, 3-10 (in Ukrainian, Russian summary).

D. G. Mejzler (1953), The study of the limit laws for the variational series, Trudy Inst. Mat. Akad. Nauk. Uzbek. SSR, no. 10, 96-105 (in Russian).

D. G. Mejzler (1956), On the problem of the limit distribution for the maximal term of a variational series, L'vov. Politekhn. Inst. Nauchn. Zap. Ser. Fiz.-Mat., no. 38, 90-109 (in Russian).

D. G. Mejzler and I. Weissman (1969), On some results of N. V. Smirnov concerning limit distributions of variational series, Ann. Math. Statist. 40, 480-491.

H. Minc (1978), Permanents, Encyclopedia Math. Appl. 6, Addison-Wesley, Reading, MA. 
H. Minc (1983), Theory of permanents, 1978-1981, Linear and Multilinear Algebra 12, $227-263$.

H. Minc (1987), Theory of permanents, 1982-1985, ibid. 21, 109-148.

R. Mucci (1977), Limit theorems for extremes, Ph.D. thesis, Temple Univ.

A. E. Sarhan and B. G. Greenberg (1962), Contributions to Order Statistics, Wiley, New York

J. Tiago de Oliveira (1976), Asymptotic behaviour of maximum with periodic disturbances, Ann. Inst. Statist. Math. 28, 19-23.

I. Weissman (1975a), Extremal processes generated by independent non-identically distributed random variables, Ann. Probab. 3, 172-177.

I. Weissman (1975b), On location and scale functions of a class of limiting processes with application to extreme value theory, ibid., 178-181.

Department of Mathematics

Faculty of Science

Zagazig University

Zagazig, Egypt

E-mail: hbarakat2@hotmail.com

Received on 12.3.2001;

revised version on 11.3.2002 